ISBN 978-93-86878-12-0

9th International Conference on Urban Planning, Architecture, Civil and Environment Engineering

(UPACEE-18)

Kyoto (Japan) April 17-18, 2018

\title{
Japanese T-House Project
}

\author{
Berrak Balci ${ }^{1}$, Michael Edward Young ${ }^{2}$ and Omer Caparti ${ }^{3}$ \\ ${ }^{1} \mathrm{MSc}$, Department of Architectural Engineering, Politecnico di Milano, Italy \\ ${ }^{2}$ Lecturer, Department of Architecture, Izmir University of Economics, Izmir, Turkey \\ ${ }^{3}$ Lecturer, Department of Architecture, Izmir University of Economics, Izmir, Turkey
}

\begin{abstract}
This project was a part of a course of Bachelor of Architecture at the Izmir University of Economics, Turkey. The project site is located in a neighborhood of Izmir. The conceptual design attempts to redefine the Japanese culture in terms of 'gathering'. When the concept of 'sharing' is taken into account, the 'C form' had been thought. The form of the T-House comes from the idea of an inviting and gathering concept. For this reason, this shape is repeated several times, which provides an indication of invitation to the guests. Furthermore, in the conceptual phase, the casting of stucco on the ground of the site is analyzed. Some layers such as 'table', 'roof', 'earth' and 'fire' appeared and differentiated. Accordingly, the percentage of them is determined and these elements are applied to the spaces in the building. The spaces of this T-House attempts at combining the traditional elements of Japanese culture with the modern architectural representations. Moreover, there is also a courtyard designed in this unit to grow vegetables, which is a significant area of Japanese culture. The users of this building are also able to watch the view to the courtyard full of greenery from the inside. There are also other several unique elements of Japanese architecture used to design this project. Considering the importance of joinery techniques of Japanese carpenters, the joints in the project had been designed accordingly.
\end{abstract}

Keywords: architectural design, Japanese T-House, traditional elements, vegetation

\section{Introduction}

This project, being part of an undergraduate degree programme, is a design project of the architectural studio course in Izmir University of Economics, Turkey. The project site is located at the territory of Izmir, Turkey. As the undergraduate students, in this project, it is expected to design a Japanese T-House building in this area.

\subsection{Preliminary Research}

The deriving idea behind the design started from the research of the works of the famous architect, Louis Kahn. He believes that how the nature keeps the changes around it, people also record the stories around them. According to him, this expression is called ' $a r t$ '. Moreover, he was also interested in the horizon line between the sky and the sea. Since the project site in Izmir is located at the seaside, the works of Kahn are investigated during the preliminary stage of this project.

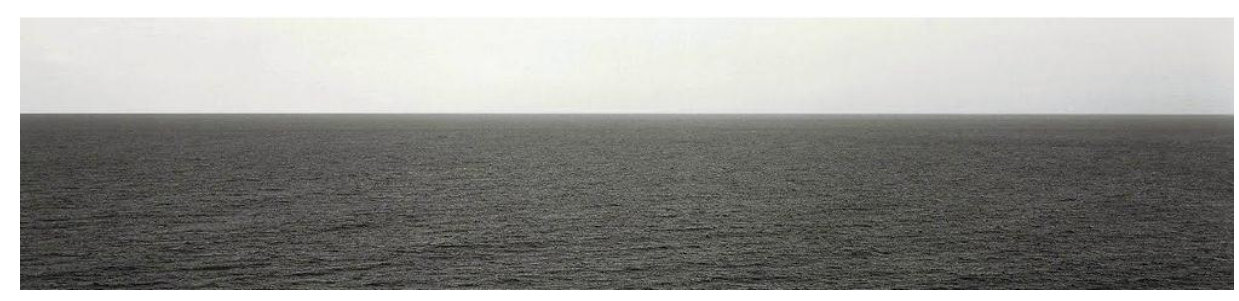

Fig.1: The horizon 


\subsection{Site Analysis}

Before the design of the T-House, an investigation about the site is made. In order to achieve this goal, the site is observed through several methods. One is the observation through senses at the site. The other is physical observation of the site. The latter is achieved after preparing the plaster and the foam on the site. First of all, the foam is prepared and put onto the soil at the site. Then, the plaster is prepared and poured directly into the foam on the soil. After a few days, the students are supposed to visit the site on order to see whether the plaster is dry enough to be removed from the soil. When it is dry, it is removed and brought into the studio class. Later, the analysis phase of the plaster occurred. The images below (Fig.2) represent several patterns after casting.
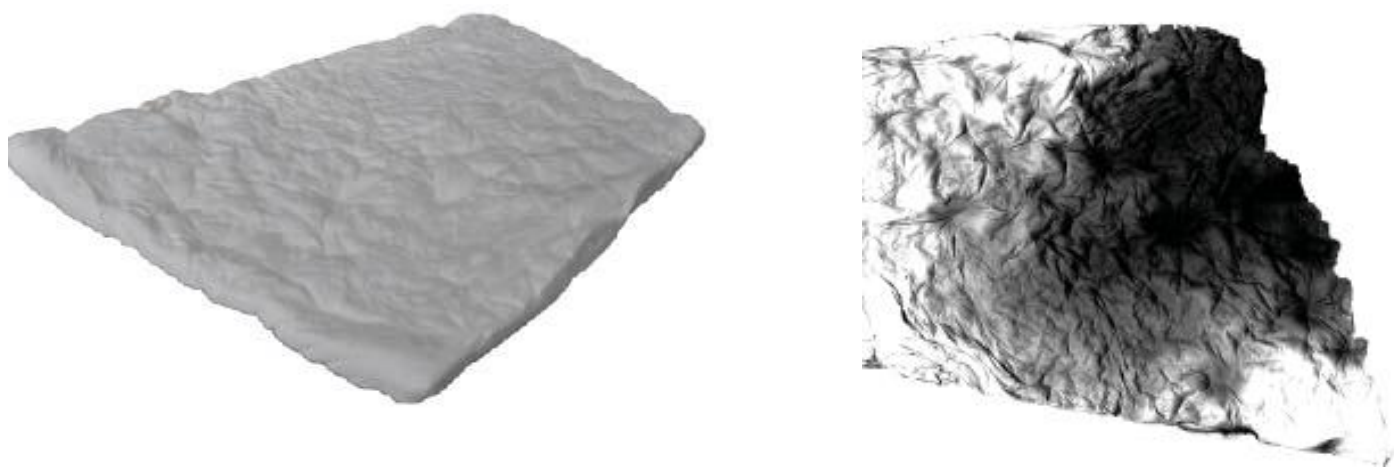

Fig.2: Pattern on the plaster

The images below (Fig.3) show some preliminary works of the pattern on the plaster. The patterns on each of them are analyzed in terms of different materials, organization and layout. The elements such as branches, soil, leaves and even tiny animals on the plaster are related with each other at the conceptual phase in order to create the spaces of the T-House building. The images below (Fig.3) are the works done in Photoshop.
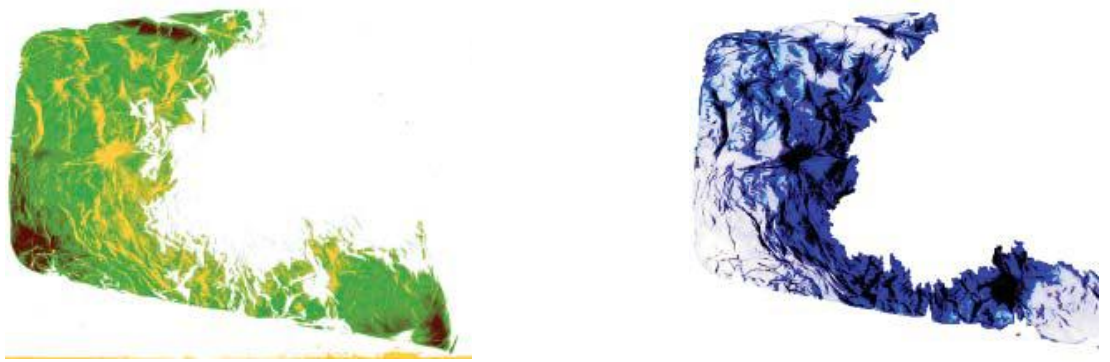

Fig.3: Initial studies of the pattern

The images below (Fig.4) represent the works by sketches. While the works above (Fig.3) represent more cluster organizations, the sketches below are more linear. At the design phase of the project, the cluster ones are used when grouping the host, guests and visitors at the building for a tea ceremony. On the other hand, the circulation of the building is taken from the idea of linear organization as depicted below.
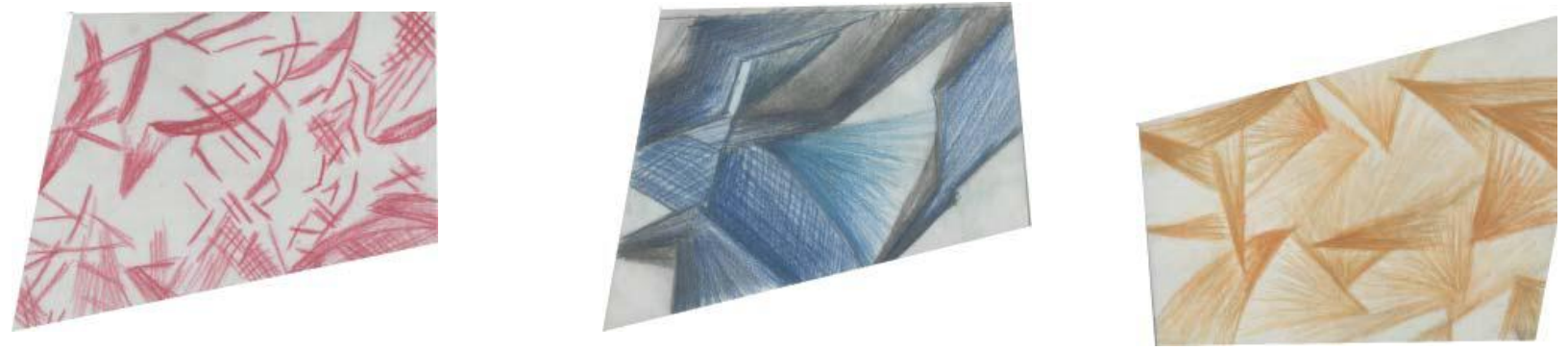

Fig.4: Sketches of the pattern 


\subsection{Japanese Tea Houses}

In Japan, the tea houses are essential buildings. The crucial part is to use the space as a ritual of dining which means relaxing. As also the name states, while dining, there is a ritual of drinking tea in these buildings. In fact, it is called as 'tea ceremony' to hold a gathering event. The images below are examples from tea houses at Tojiin Temple in Kyoto, Japan.
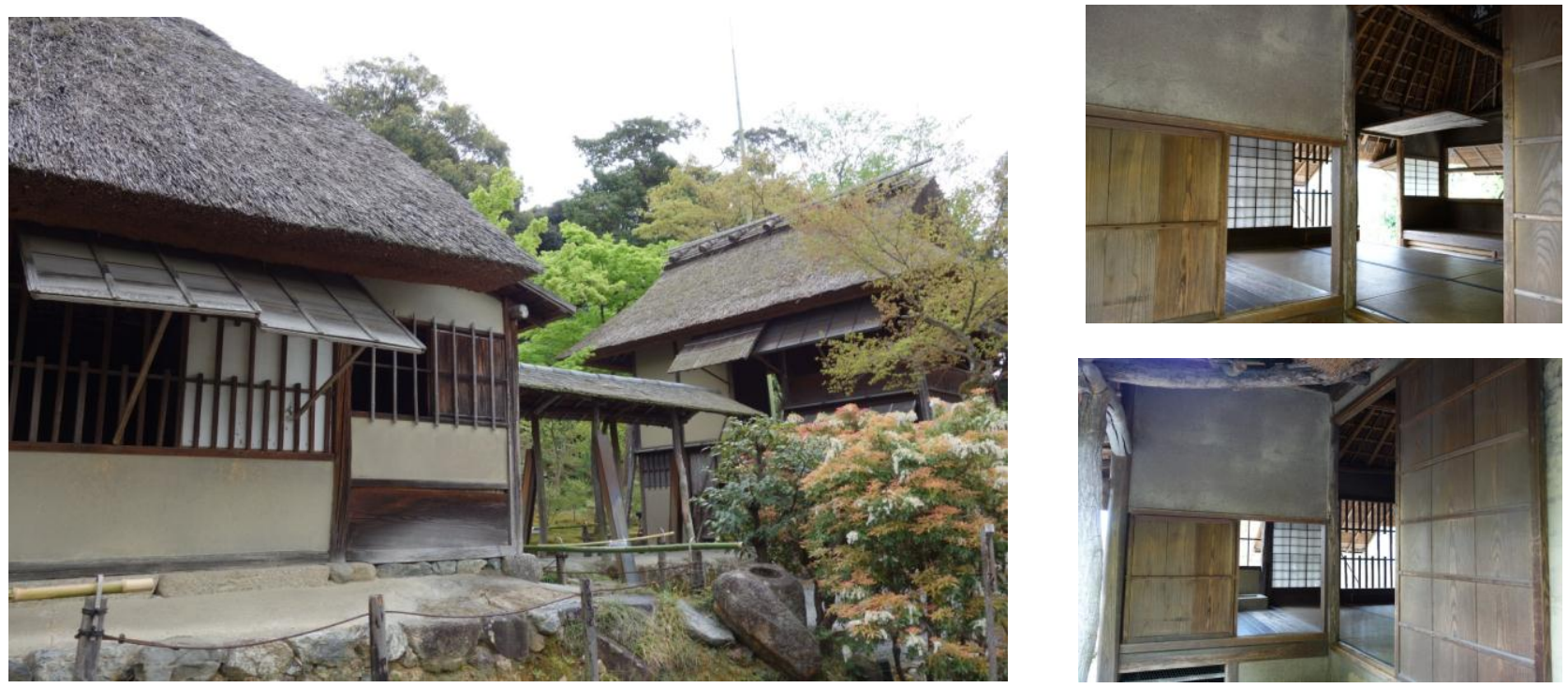

\section{Concept}

Fig.5: Tea Houses in Kyoto, Japan

The design concept of the project derives from the idea of relating the spaces appropriate to use and equally to the site. How these spaces are related with one another after casting the plaster on the soil are essential at this phase. More importantly, how these spaces can be realized through architecture is crucial. Not only with the walls, but architecture engages multiple senses of people, thus the feelings of different types of the users are considered.

There are three kinds of users in this Japanese T-House building. These are the host, guests and visitors. Thus, this building is a symbiotic relationship between these users (1). The images below (Fig.6) represent the parts of the building conceptually. The form of the building is modelled physically. When it comes to serve the guests, or sharing an event with the visitors, it is kind of 'gathering'. For this reason, the 'C shape' which indicates union, being together and more than that, welcoming form for the building, is selected.
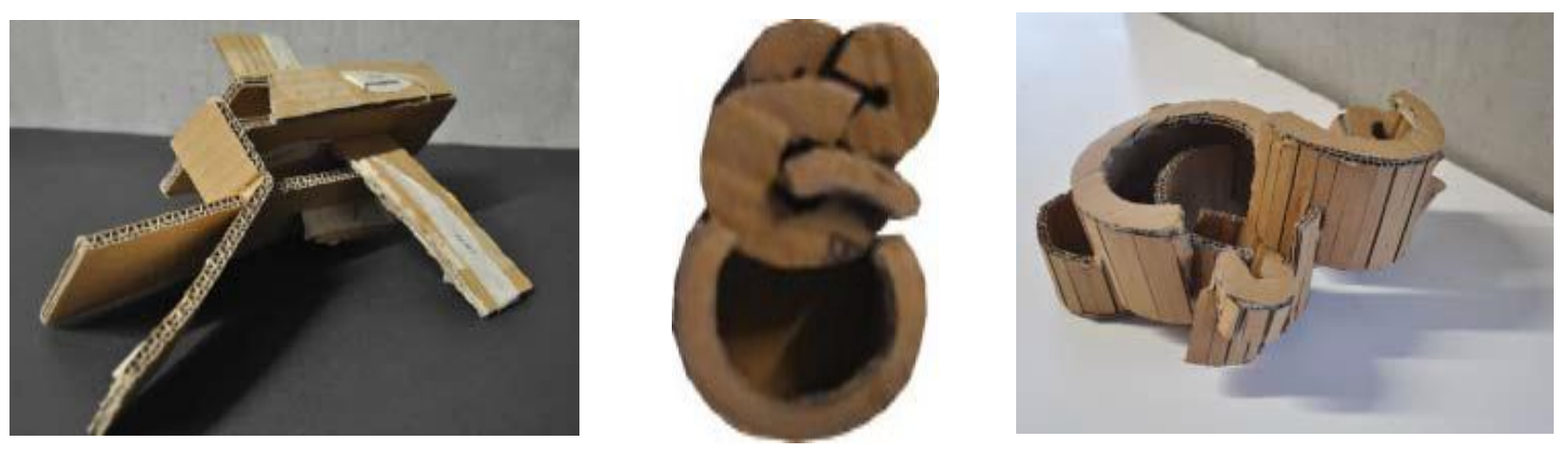

Fig.6: Physical models of the design 
When designing the spaces according to their functions, the purpose of host, guests and visitors should be considered. Guests usually visit the place once while the visitors make periodic use of the facility. The physical models below (Fig.7) indicate the preliminary models of the design. The intersections of the elements of the model on the left are related to the intersections of the several materials on the plaster after casting.
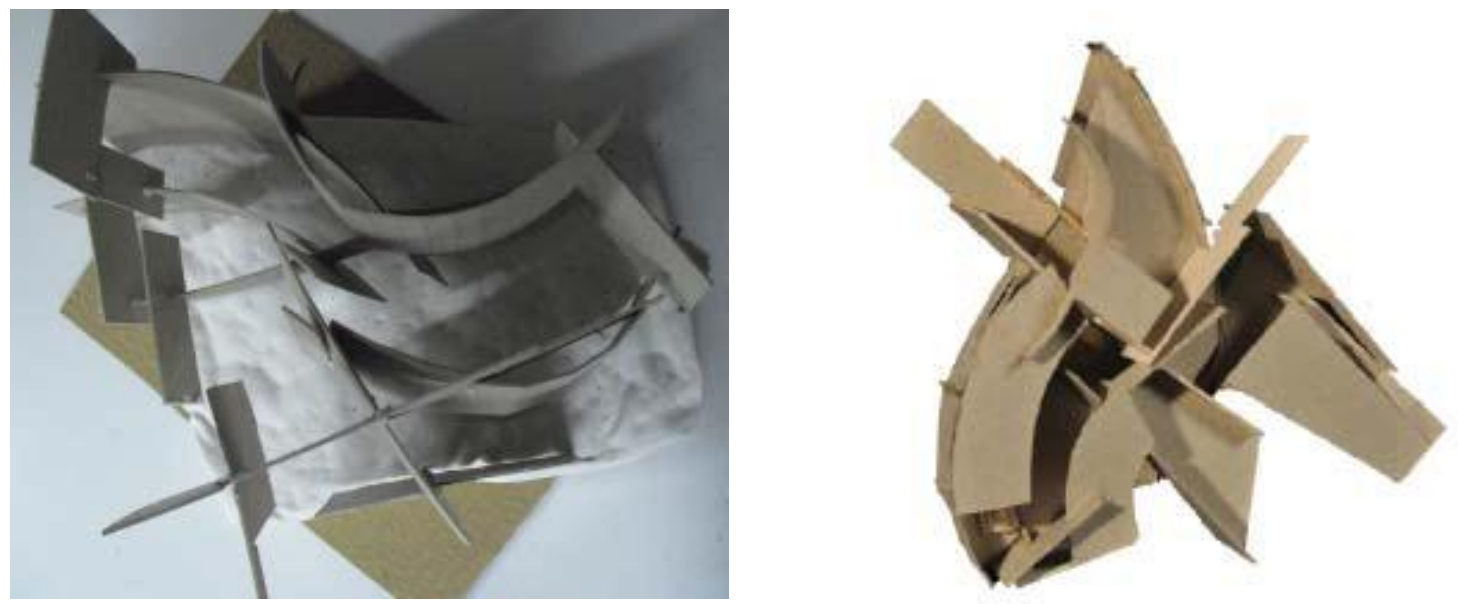

Fig.7: Physical models

The programme of the T-House building refers to the indoor and outdoor spaces. The indoor units are rooms for private and communal meals, meditation room, food storage and preparation area and living spaces. On the other hand, the outdoor spaces are mainly the courtyards to grow vegetables and engage the visitors with agriculture.

The drawing on the left side below indicates the units, especially the courtyards as open spaces. This space is used for the events to share the fruits and vegetables with the guests and the visitors. Furthermore, in this project, local materials are intended to be used. These are wood and bamboo especially used in the garden.
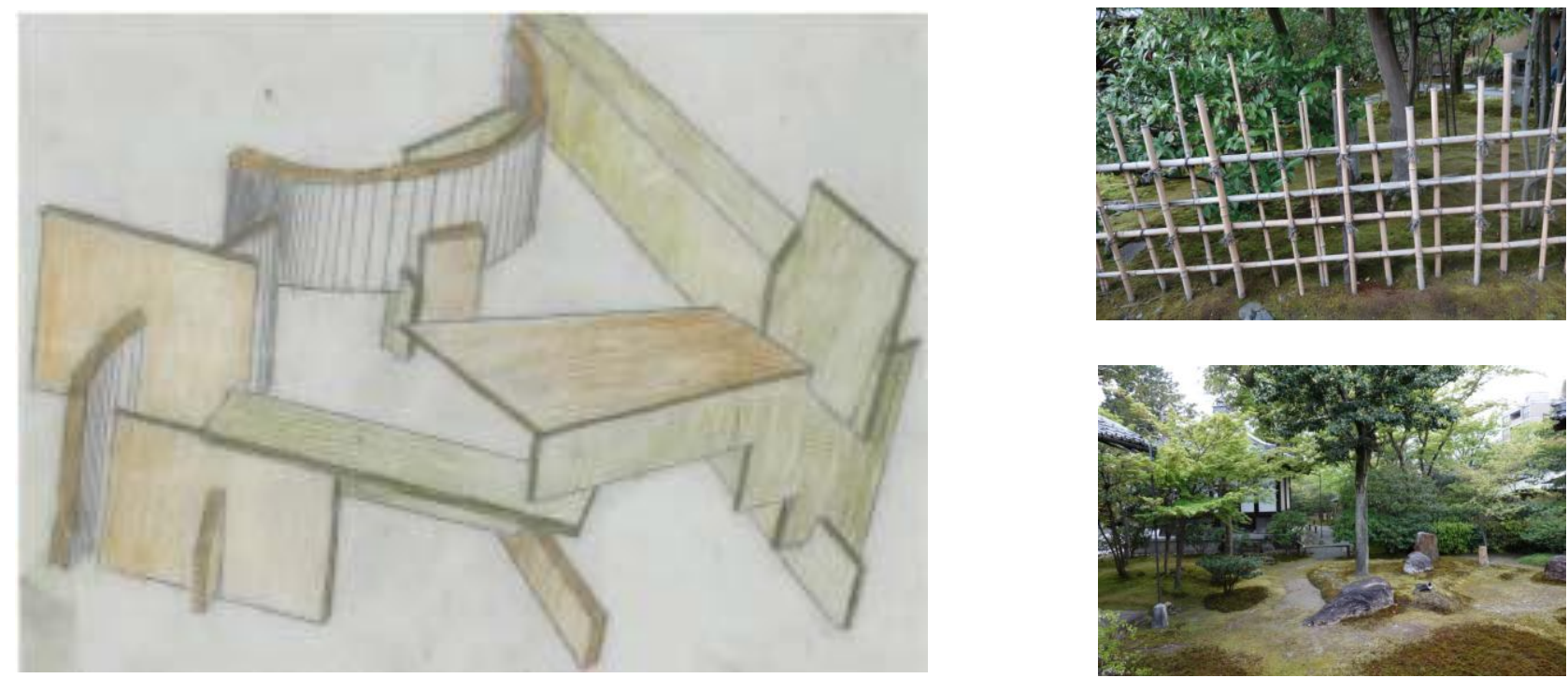

\section{Final Design}

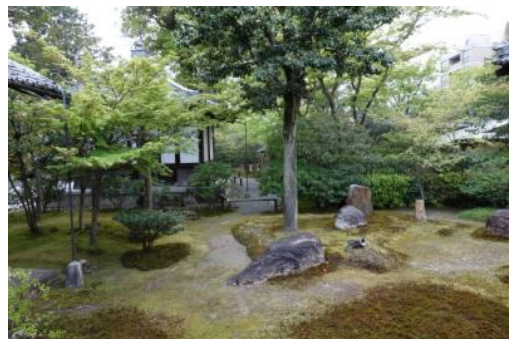

Fig.8: The concept

In the final design stage, the spaces in the building are constructed considering fluidity and flexibility. It is also thought to be the target of gathering and welcoming activities. In order to welcome to the guests and the visitors, the form of ' $\mathrm{C}$ shape' is considered for a friendly environment. Moreover, in the sections below, the 
courtyard is easily noticeable. This space is not only to share the event of tea ceremony, but also to grow food. When the visitors come to this area, they are able to plant food and collect it when they are mature.
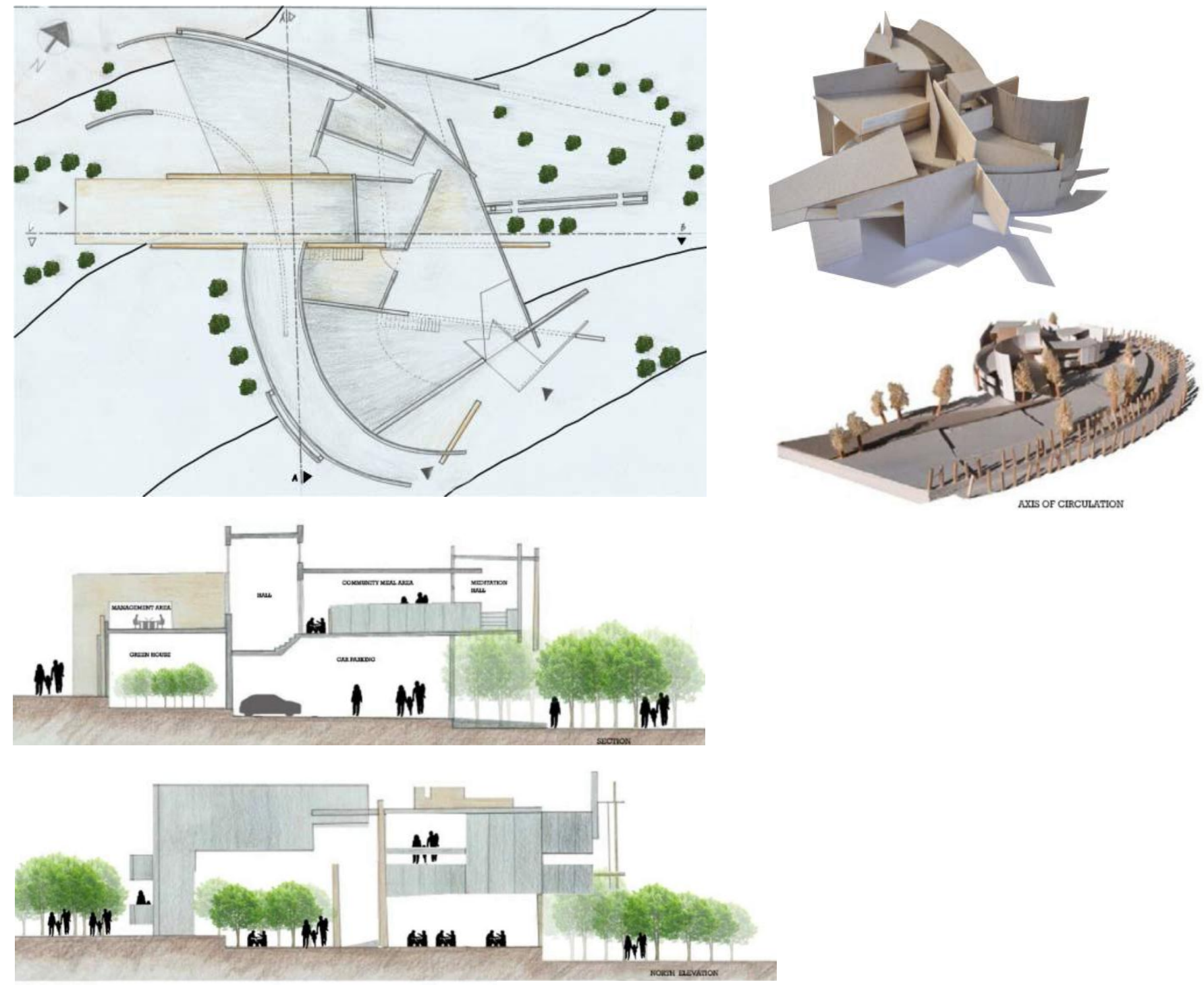

Fig.9: Final drawings and models

The design of the entrance of the T-House building comes from the concept of the entrances of Japanese tea houses. Since bamboo is commonly used in the gardens, the entrance is designed with the local material, bamboo and wooden boundaries.

\section{Conclusion}

This project aims at designing a territory according to the Japanese tea ceremony ritual in Izmir, Turkey. After extensive research on Japanese tea house buildings, this project is designed in consideration with the spaces and activities required. One of the crucial elements is to design the gardens in this project. Since Japanese gardens merge several activities as rituals and ceremonies, the outdoor spaces as courtyards are as essential as indoors.

\section{Reference}

[1] M.E.Young, O.Caparti, "T-House," ARCH 301 Architectural Design, Izmir University of Economics, Izmir, Turkey. 2011 